\title{
The Fresnel Imager: learning from ground-based generation I prototype
}

\author{
Denis Serre · Laurent Koechlin • Paul Deba
}

Received: 12 February 2010 / Accepted: 5 September 2010 / Published online: 22 September 2010 (C) The Author(s) 2010. This article is published with open access at Springerlink.com

\begin{abstract}
The "Generation I Fresnel Imager Prototype" is a ground-based prototype of a Fresnel Imager (Koechlin et al., Astron Astrophys 443:709$720,2005)$, reduced in size (optical elements have a dimension of a few $\mathrm{cm}$ ) but which features all the elements of an operational Fresnel Imager, and integrated in a clean room. Its design has started in October 2004, the first images were obtained early 2006, the key optical element: a cophased Fresnel Zone Lens, was integrated during summer 2006, and since then it has been used to evaluate new types of target shapes, of Fresnel Array cuttings... . It has been decommissioned end of 2008, some constitutive elements becoming parts of the so-called "Generation II Fresnel Imager Prototype". We present the constitutive elements and the achieved results, and the lessons learned from this prototype.
\end{abstract}

Keywords Fresnel • High dynamic range • High angular resolution • Ground-based prototype

\section{Introduction}

The Fresnel Imager is a concept of high angular resolution and high photometric dynamic range space-based telescope, featuring two satellites flying in

\footnotetext{
D. Serre $(\varangle)$

Leiden Observatory, Leiden University, PO Box 9513, 2300RA Leiden, The Netherlands e-mail: serre@strw.leidenuniv.nl

L. Koechlin · P. Deba

Laboratoire d'Astrophysique de Toulouse-Tarbes, Université de Toulouse, CNRS,

14 Avenue Edouard Belin, 31400 Toulouse, France
} 
formation. The first satellite holds a binary optics whose design is close to that of a Fresnel (or Soret) Zone Plate (FZP), which focusses a fraction of the light (typ. 4-10\%) with a focal length $\propto \frac{1}{\lambda}$. The advantages of the concept are the highly relaxed manufacturing tolerances to get a given wavefront quality, the high angular resolution achievable, and the potential high photometric dynamic range (see Koechlin et al. [2] for a review, or articles from this Experimental Astronomy special issue.). The second satellite holds a focal module, the design of it leading to a rigourous chromatic correction. The F-number of the FZP being really high (typ. more than 300), the focussing and focal modules must be separated on two satellites flying in formation.

Not only working on the concept, at Laboratoire d'Astrophysique de Toulouse-Tarbes we have also developed prototypes of this Fresnel Imager. The first of them was aimed to prove that the concept is actually working, therefore besides simply producing images showing that the achromatization is effective, that the angular resolution is limited by the size of the FZP only, and that a high dynamic range can be achieved. The design of this "Generation I Fresnel Imager Prototype" started end of 2004, and the validation was fully done at end 2006. It has since been used to test various potential improvements, and has been decommissioned late 2008. The preliminary and main results have been presented in Serre et al. [3, 4], therefore after reminds in Sections 2 and 3 of the constitutive elements and main results, Section 4 highlights some of the critical lessons that we have learnt from this GenI prototype.

\section{Constitutive elements}

The prototype can be split into three main modules: a module creating a source rejected at an infinite distance, the FZP (or Fresnel Array) module, and the focal module, the latest two being the Fresnel Imager prototype in itself. Figure 1 presents the optical scheme involved in the two last ones, and the following subsections briefly present its implementation on the prototype. Detailed information on the demonstration of the achromatization scheme and the design of the thereafter called "diverging cophased (or blazed) Fresnel Zone Lens" can be found in Serre et al. [4].

\subsection{Fresnel Arrays}

Two different Fresnel Arrays have been tested (Fig. 2): the first one was involving an orthogonal geometry of the zones therefore rectangular holes, whereas the second one is basically constituted of a standard Soret zone plate (concentric rings) onto which is surimposed a matrix of vertical and horizontal bars sustaining the whole structure, those bars being placed at the Fresnel zones themselves in order to alter the image quality as minimal as possible. With the second Fresnel Array, the quantity of light in the central core of the PSF is typ. 50\% higher, and the spikes are also fainter. Apart from these 


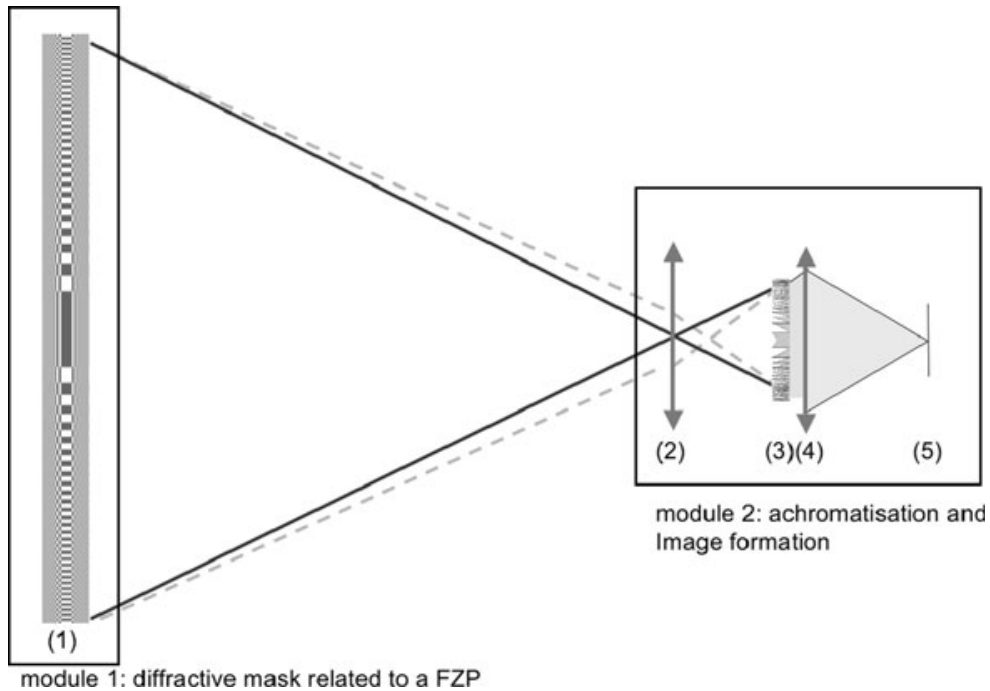

Fig. 1 The Fresnel Array placed in plane (1) focusses different wavelengths at different distances. In the second module, an achromatic field optics in plane (2) forms a pupil plane (3), where the blazed Fresnel Zone Lens (FZL) is placed. The chromatic correction induced by this scheme is rigorous [5], and the emerging beam divergent. An achromatic lens (4) is placed to make it converge. The achromatic image is formed onto plane (5)

different cuttings, the two arrays are similar in dimensions ( $8 \mathrm{~cm}$ side), number of Fresnel zones (116 from center to corner, meaning for example 26,680 holes in the Fresnel Array with the orthogonal geometry), and therefore focal length (23 m @ $600 \mathrm{~nm}$ ). They have both been carved with a UV laser in thin metal
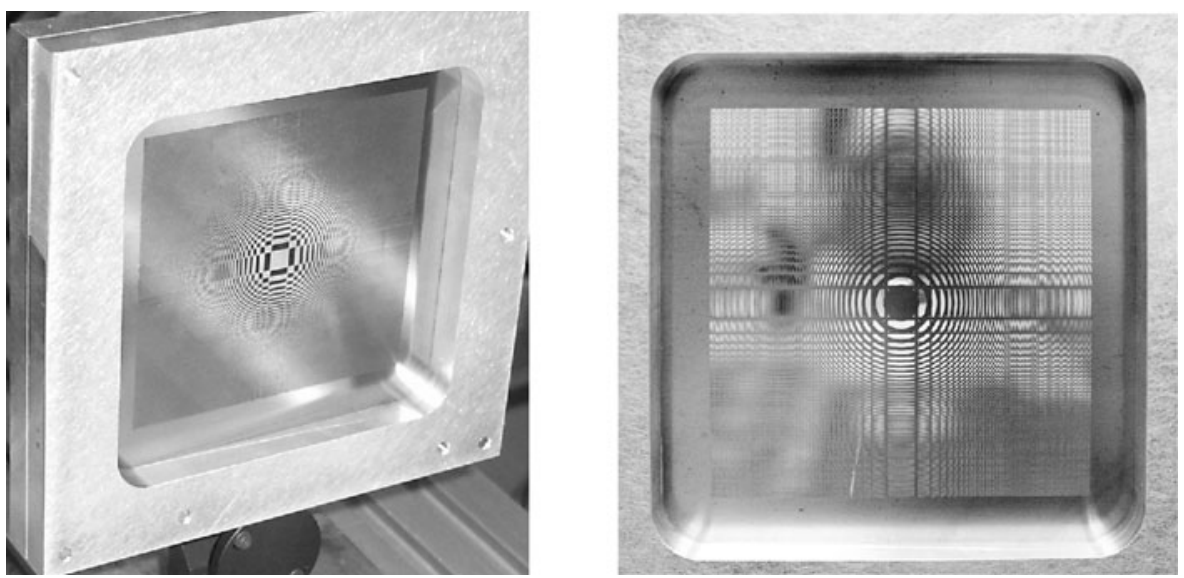

Fig. 2 Left first carved Fresnel Array, involving an orthogonal geometry. Right second carved Fresnel Array, involving a circular geometry plus a 'multispider' sustaining the zones. Both Fresnel Arrays have a $8 \mathrm{~cm}$ side and feature 116 Fresnel zones from center to corner 


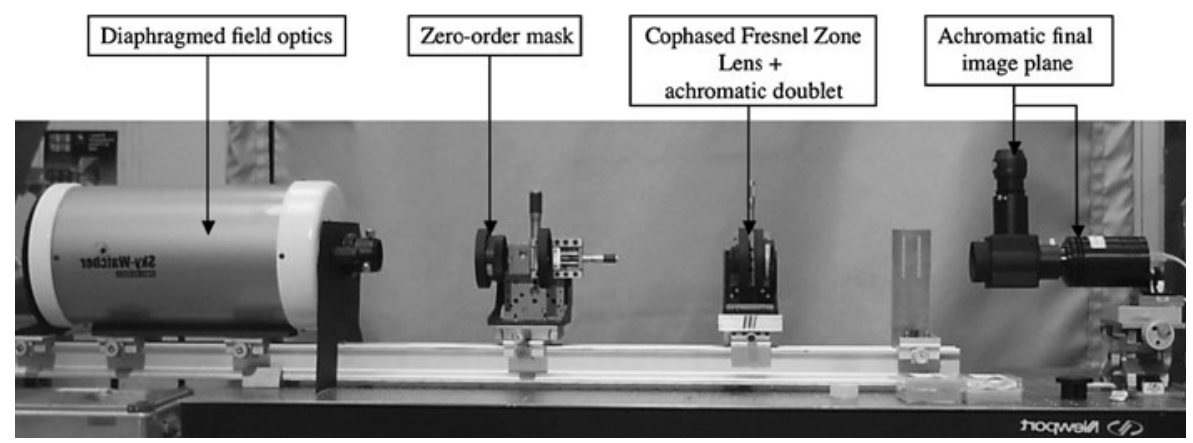

Fig. 3 The focal module is composed of a (diaphragmed) Maksutov telescope used as a field optical element, a cophased diverging Fresnel Zone Lens placed in a (pupil) plane conjugated to that of the Fresnel Array by the Maksutov, and an achromatic doublet next to that pupil plane. The achromatic image plane can be directed either toward a CCD camera or onto an eyepiece for direct visualization. Additionally, a mask is placed at the focal plane of the Maksutov in order to eliminate the residual light from the 0 and -1 diffraction orders of the Fresnel Array

foils $(80 \mu \mathrm{m}$ thickness inox for the first one, $50 \mu \mathrm{m}$ thickness $\mathrm{CuBe}$ for the second one).

\subsection{Focal module}

The whole problematic of the focal module is to image the Fresnel Array onto a cophased diverging Fresnel Zone Lens, the Fresnel zones of the first one being surimposed to those of the second, as demonstrated in Koechlin et al. [1]. The integrated focal module can be seen on Fig. 3 and is a direct application of the optical scheme as presented on Fig. 1: one can recognize the fields optics (a Maksutov telescope diaphragmed to a $3 \mathrm{~cm}$ diameter), the cophased Fresnel Zone Lens (3D view of the central zones on Fig. 4), the image

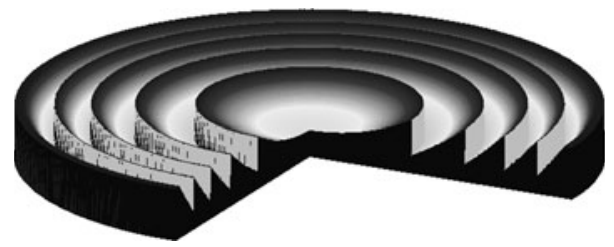

Fig. 4 3D view of the five central zones of a diverging FZL. The vertical scale is highly exaggerated, as the depth of the slopes is $1.31 \mu \mathrm{m}$ in the case of our GenI prototype, whereas the radius of the central zone is $600 \mu \mathrm{m}$ and the width of the most external zone is $\sim 38 \mu \mathrm{m}$. The wavelength for which the design has been optimized $\left(\lambda_{\text {blaze }}\right)$ is $600 \mathrm{~nm}$. The FZL of effective diameter $16.054 \mathrm{~mm}$ has been carved by SILIOS technology onto a flat window using photolitography technics. The detailed explanation of the obtention of the profile and the computed efficiency can be read in Serre et al. [4] 
reformation achromatic optical element (a doublet), and the achromatized image plane.

\subsection{Source module}

The source module consists of various light sources (halogen, plus potentially $50 \mathrm{~nm}$ bandpass filters, and LEDs) and various target shapes (pinholes, homogeneous discs, USAF target, 'galaxy' shaped target, but also output of monomode optical fibers) set at the focal plane of a parabolic mirror. The mirror is rejecting the target at an infinite distance. The emerging beam then falls onto the Fresnel Array, marking the start of the Fresnel Imager prototype in itself.

\section{Results}

As formerly presented in Serre et al. [3, 4], the angular resolution of the GenI prototype is limited by the size of the Fresnel Array at all the wavelengths (for wavelengths which are not diaphragmed by the field optics), therefore the achromatisation scheme is validated: there is no longitudinal nor transversal chromatic aberration. The dynamic range is close to the predictions done by the Instrument Numerical Model (see other article from Serre in this Experimental Astronomy special issue). A summary of the results can be seen on Figs. 5 and 6.
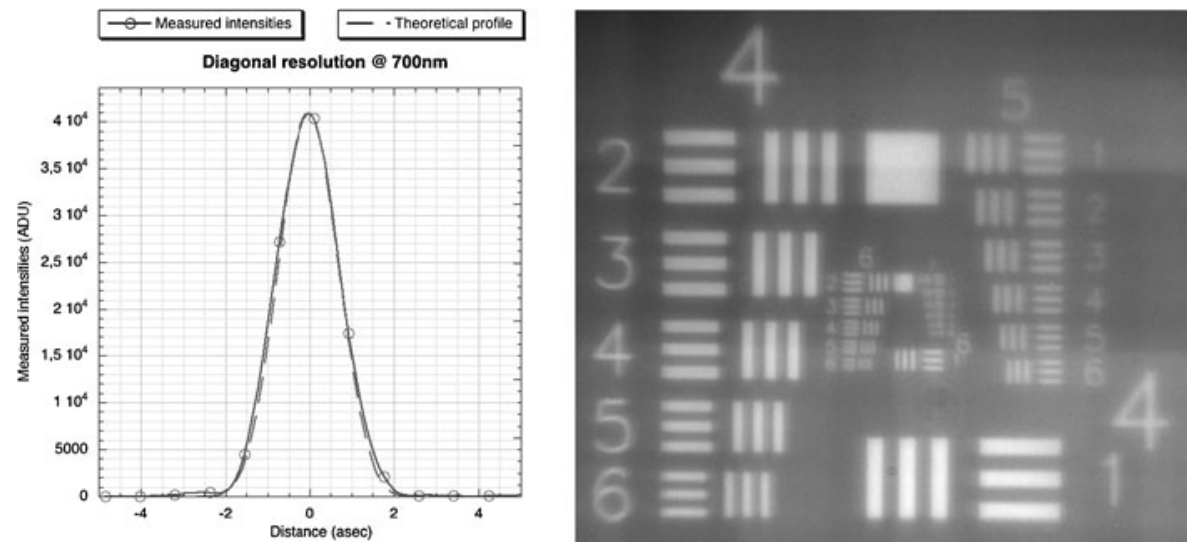

Fig. 5 Left comparison between the theoretical and measured angular resolution, using as target a pinhole illuminated by an halogen lamp filtered by a $50 \mathrm{~nm}$ bandpass filter centered on $700 \mathrm{~nm}$ (therefore at a wavelength different of $\lambda_{\text {blaze }}$ ). Right standard USAF target illuminated by a white led, as seen by the Fresnel Imager. The ' 6 ' group number associated to the ' 4 ' element number presents a number of line pairs per mm corresponding to the diffraction limit of our prototype, validating here also the angular resolution. For information, the image is only dark-substracted, and no threshold has been applied 

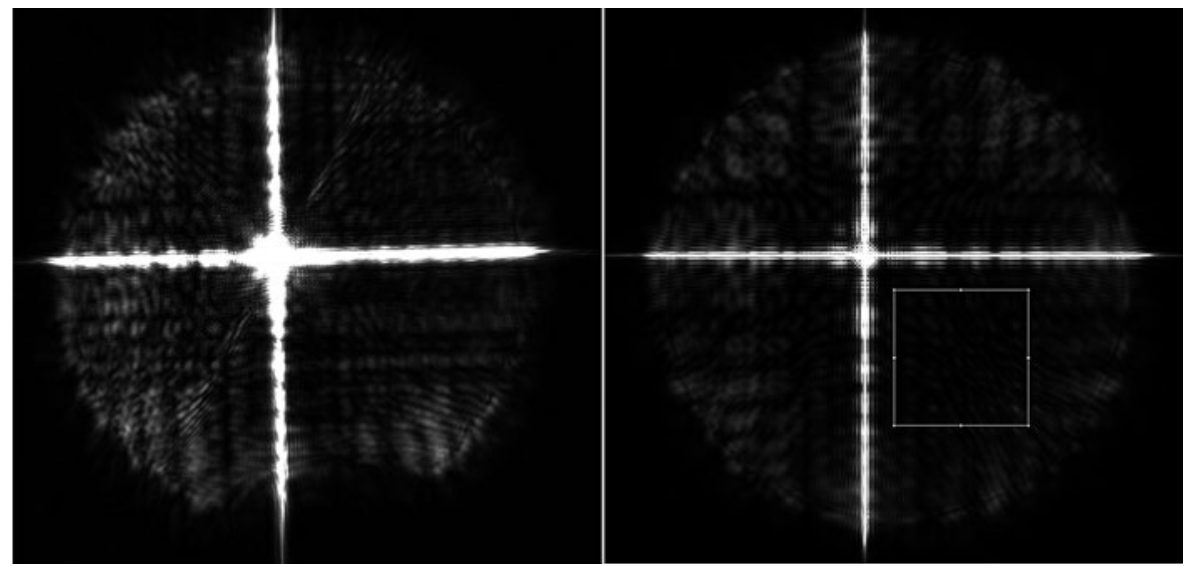

Fig. 6 Left PSF obtained using as target a monomode fiber iluminated with a luxeon LED (peak emission at $630 \mathrm{~nm}$ ), simply dark-subtracted. Right PSF from the corresponding computer simulation. The two images are (highly) saturated with the same thresholds, in order to show the faint levels of the PSF. The dynamic range in the two cases are comparable $\left(1: 10^{-6}\right.$ in the square delimitation shown on the right figure, $2: 10^{-6}$ on the corresponding one on the real measurement, assuming 'dynamic range' as the ratio between the mean level of the delimited region and the maximum of the PSF)

\section{Learning}

Besides validating the concept of Fresnel Imager itself as briefly summarized in the previous section, this GenI prototype has taught us some lessons which are presented in the subsections below.

\subsection{Critical role of the Fresnel Zone Lens}

This GenI prototype has confirmed that, as expected, on the one hand the optical scheme presented on Fig. 1 actually works, and on the other hand that the use of a cophased FZL in a pupil plane, if not necessary because a binary optical element can be used, greatly improves the efficiency (Fig. 7).

\subsection{Manufacture capabilities}

We have also learnt some issues about some of the state-of-the-art technology, especially about the manufacturing or carving of binary and phase masks, the cophased Fresnel Zone Lens being nothing else than a phase mask. What can be seen on Fig. 8 is an image of the central zones of the FZP used to obtain the left image of Fig. 7, but this time set as the target of the Fresnel Imager and observed with the nominal Fresnel Imager, including the cophased FZL. This acquisition permitted us to estimate that the UV laser carving technology provides a roughness of $\simeq 10$ microns on the cutting of edges.

Some recent and preliminary analysis of the manufactured cophased FZL using Scanning Electron and Atomic Force Microscopes (SEM and AFM) 

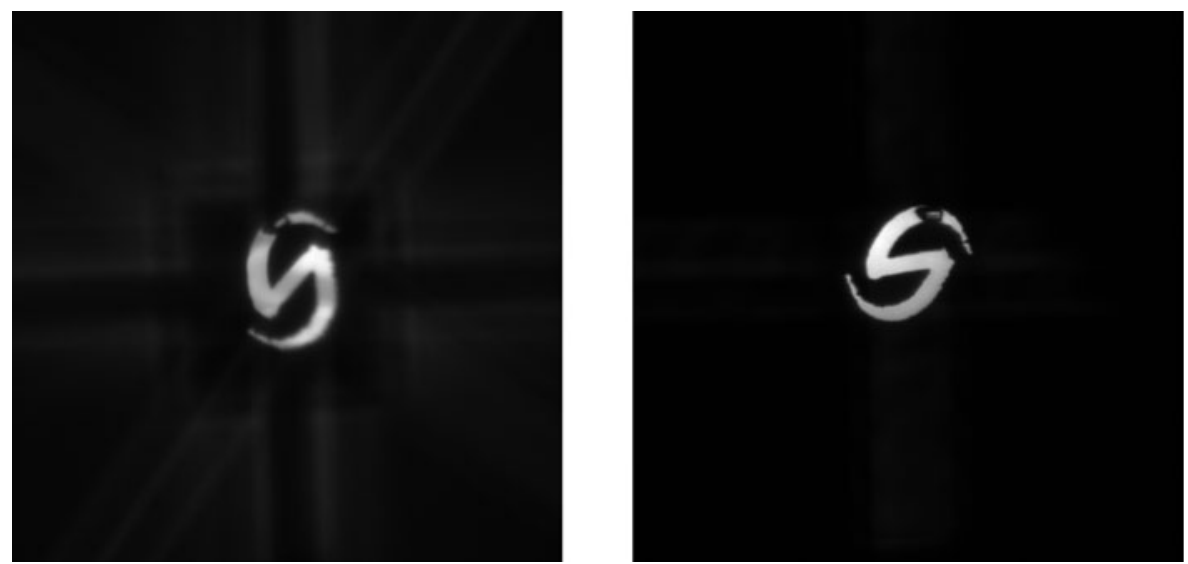

Fig. 7 In the left and right acquisitions, the target observed was a 'galaxy' carved in a metal foil illuminated by an halogen source. The image on the right is the image obtained with the instrument configuration presented previously, including the cophased Fresnel Zone Lens, but the image on the left was obtained earlier, before having ordered the manufacturing of the FZL. Instead of the FZL, we had placed in the pupil plane a binary Fresnel Zone Plate, basically an homothetic copy of the Fresnel Array presented in Section 2.1 (same number of zones), but with a side size being $11 \mathrm{~mm}$ instead of $8 \mathrm{~cm}$. This FZP of course had a -1 order of interference, therefore we indeed get an image of the target on the CCD camera, but the efficiency of the FZP at order -1 being low (few \%), on the one hand the image of the target was much fainter than that obtained with the FZL set in place, and on the other hand the quantity of background (unfocussed) light is much higher. One can also see on the left image the shadow of the order 0 mask, and also diagonal spikes due to the orthogonal geometry of the FZP, whose axis were rotated with respect to those of the front Fresnel Array. Finally the image quality (angular resolution) of the right image is better than that of the left image because the manufacturing of the most external (and therefore really small) zones of the FZP was a challenge (see Fig. 8)

have shown among other information that the micro-roughness of the surface is stronger at the bottom of the slopes than at the top, which can be understood as the original material is a flat window and the manufacturing technic is photolitography: the deeper the window has to be engraved, the higher the number of masks will be used, therefore the higher the number of expositions will be. However, for this GenI prototype, the difference in dynamic range with respect to the INM simulations is faint (Fig. 6), and could be attributed to various other reasons. But, for future applications requiring high dynamic range, this aspect should be investigated.

\subsection{Effect of pupils misalignment}

Already before the implementation of the cophased FZL, we could notice simply through the eyepiece that if the image of the Fresnel Array was not 'perfectly' surimposed to the binary FZP, we could see the spectrum of the source (Fig. 9).

We assume that $c$ is the size of the two pupils, supposed common, and $N$ is the number of Fresnel zones from center to edge. We also introduce $d$ as the 
Fig. 8 We used the Fresnel Zone Plate used prior to the cophased Fresnel Zone Lens as a target for the Fresnel Imager. Irregularities of cutting by the UV laser on this specific material ( $50 \mu \mathrm{m}$ thickness $\mathrm{CuBe}$ ) can be seen on the edges of the zones. The width of the rectangular aperture on the top right of the picture (6th aperture along one of the two axis of the FZP, over 58) is $77 \mu \mathrm{m}$

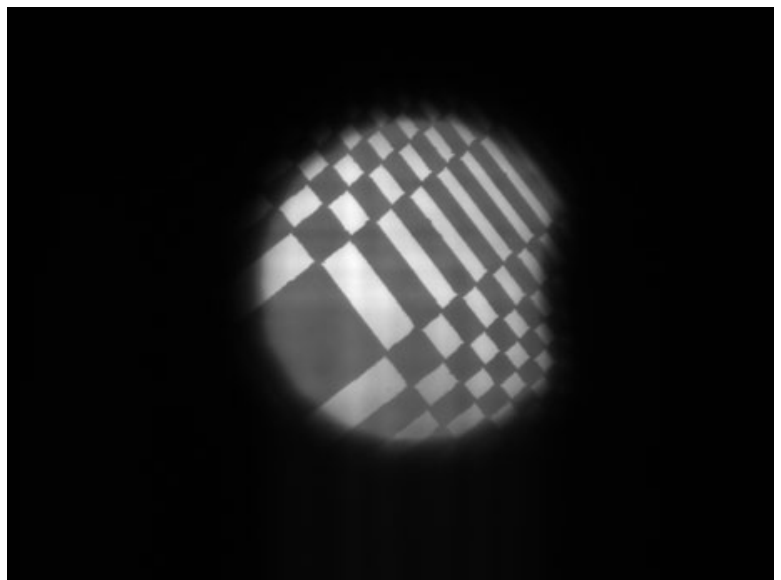

distance of a point of the pupil plane to its center, and $\Delta d$ the shift between the centers of the two surimposed pupils. The phase of the incident wavefront $\varphi_{i}$ at position $d$ and the phase $\varphi_{c}$ added by the corrective FZL (or FZP) at order -1 can then be written (Fig. 10):

$$
\begin{aligned}
& \varphi_{i}=2 \pi N\left(\frac{2 d}{c}\right)^{2} \\
& \varphi_{c}=-2 \pi N\left(\frac{2(d-\Delta d)}{c}\right)^{2}
\end{aligned}
$$
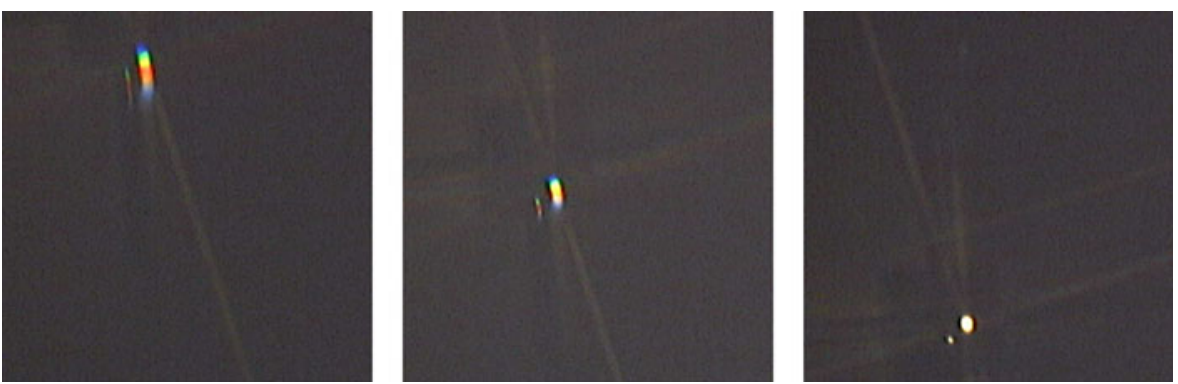

Fig. 9 The target is a double source point, illuminated by an halogen lamp. As in left image of Fig. 7, because the cophased FZL was not used at this time, two sets of orthogonal spikes can be seen: one set due to the Fresnel Array, one set due to the binary FZP used to correct the chromatism. From left to right: using a micrometric translation stage, we shift the binary FZP (which was later replaced by the cophased FZL) so that the Fresnel zones of the image of the Fresnel Array produced by the Maksutov telescope are surimposed to the zones of the binary FZP. At the beginning of the process (left image), when the two pupils are shifted, one can see the (low resolution) spectrum of the target. When the process is finished (right image), the pupils are superimposed, and all the wavelengths are focused at the same coordinates of the image. A standard webcam with internal image processing capabilities was used, explaining the differences in the background luminosity and the edge effects on the bright peak. One can also notice that the ccd of the camera used is obviously sensitive to infrared wavelengths 


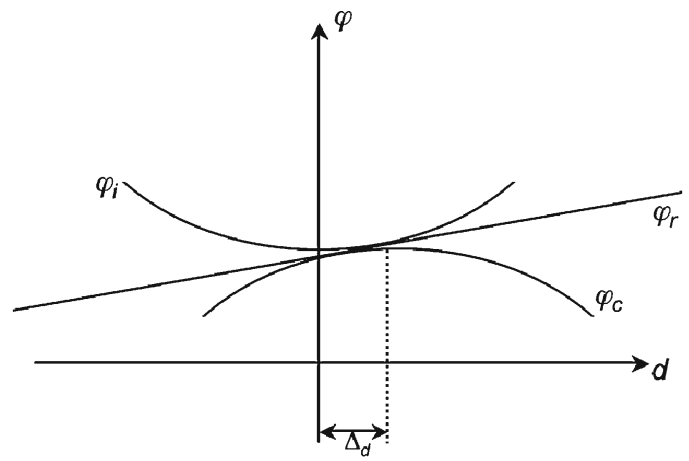

Fig. 10 If the image of the Fresnel Array is not superimposed to the corrective FZP (or FZL), the phase of the incident wavefront $\varphi_{i}$ will not be centered onto the phase $\varphi_{c}$ induced by the corrective FZP (or FZL). Therefore the emerging wavefront will be tilted, the complex amplitudes describing the wavefront having a phase linearly proportional to distance $d$ (Eq. 3 )

We retrieve $\varphi_{i}=2 \pi N$ in $d=\frac{c}{2}$, and $\varphi_{c}=-2 \pi N$ in $d=\frac{c}{2}$ if $\Delta d=0$. The resulting phase $\varphi_{r}$ can be written:

$$
\varphi_{r}=\varphi_{i}+\varphi_{c}
$$

Neglecting $\Delta d^{2}$ with respect to $d \Delta d$, we get:

$$
\varphi_{r}=2 \pi \frac{8 N \Delta d}{c^{2}} d
$$

The optical path difference at position $d$ as a function of $\lambda$ is therefore:

$$
\operatorname{opd}(d, \lambda)=\lambda \frac{8 N \Delta d}{c^{2}} d
$$

If $\Delta d=0, o p d(d, \lambda)=0$. But if $\Delta d \neq 0$, on the one hand for a fixed $\lambda$ we get $\operatorname{opd}(d, \lambda) \propto d$ : a tilt component is added, and on the other hand, this tip is proportional to $\lambda$. Therefore the global result is that what we observe on Fig. 9: a dispersion of the spectrum of the source analog to that obtained by a conventional grating having a constant step. The dispersion angle for a given $\Delta \lambda$ can be written:

$$
\tan (\Delta \alpha) \simeq \Delta \alpha=\frac{\operatorname{opd}(d, \Delta \lambda)}{d}=\Delta \lambda \frac{8 N \Delta d}{c^{2}}
$$

This dispersion angle (the differential tilt) can be compared to the size of the Airy disk $\rho$ (approximating $\rho$ to $\frac{\lambda}{c}$ ):

$$
\frac{\Delta \alpha}{\rho}=\frac{8 N \Delta d}{c} \frac{\Delta \lambda}{\lambda}
$$



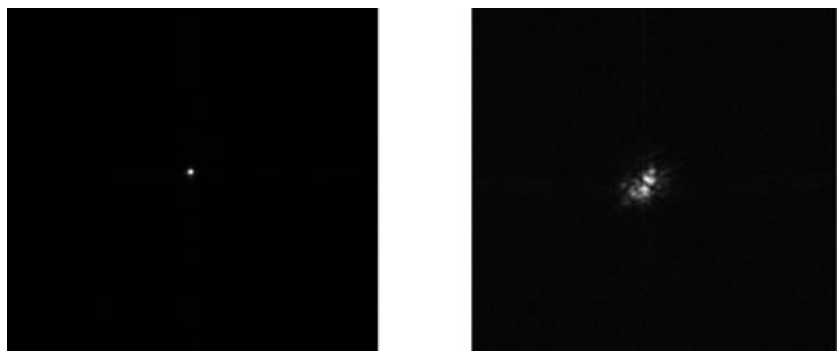

Fig. 11 Acquisitions of a pointlike source with the Fresnel Imager. Most of time we could see images close to left picture, but we also sometimes had to deal with unexpected air turbulence, as can be seen on right figure, showing a 'nice' speckle pattern

meaning that the chromatic correction will be realized if this value is below 1 ; therefore:

$$
\Delta d \leq \frac{c}{8 N} \frac{\lambda}{\Delta \lambda}
$$

One can note that as we could expect, the larger the spectral bandpass is, the stronger the superimposition constraint is. To give an idea of the values, one can notice that $\frac{c}{4 N}$ is the dimension of the most external Fresnel zone. It must also been noticed that this property can be used to obtain low-resolution spectra in non crowded fields.

\subsection{Turbulence}

Even if the prototype was set in a clean room, we sometimes had to deal with air turbulence (Fig. 11).

\section{Conclusion}

The ground-based Generation I prototype of the Fresnel Imager, now decommisionned, has experimentaly proved the efficiency of the Fresnel Imager concept: the achromatization scheme is effective, the angular resolution is limited by the size of the Fresnel Array, and the dynamic range as measured is close to the predictions of the Instrument Numerical Model. The GenI prototype has also permitted our group to be more aware of the currently available manufacturing capabilities, and has also sometimes forced us to think about the mathematical formalism which was behind some unexpected although seen results, such as the chromatic dispersion presented in Section 4.2 .

Acknowledgements The GenI Fresnel Imager prototype was funded as a R\&T contract by the Centre National d'Etudes Spatiales (CNES), with support from Université Paul Sabatier (Toulouse, France) and CNRS. Four fifth of the work presented was realized during Denis Serre's $\mathrm{PhD}$ thesis, which was co-funded by Thales Alenia Space and Europe. 
Open Access This article is distributed under the terms of the Creative Commons Attribution Noncommercial License which permits any noncommercial use, distribution, and reproduction in any medium, provided the original author(s) and source are credited.

\section{References}

1. Koechlin, L., Serre, D., Duchon, P.: High resolution imaging with fresnel interferometric arrays: suitability for exoplanet detection. Astron. Astrophys. 443, 709-720 (2005)

2. Koechlin, L., Serre, D., Deba, P., Pelló, R., Peillon, C., Duchon, P., Gomez de Castro, A.I., Karovska, M., Désert, J.-M., Ehrenreich, D., Hebrard, G., Lecavelier Des Etangs, A., Ferlet, R., Sing, D., Vidal-Madjar, A.: The Fresnel interferometric imager. Exp. Astron. 23, 379-402 (2009)

3. Serre, D., Koechlin, L., Deba, P.: Fresnel interferometric arrays for space-based imaging: testbed results. In: MacEwen, H.A., Breckinridge, J.B. (eds.) UV/Optical/IR Space Telescopes: Innovative Technologies and Concepts III. Proceedings of the SPIE, vol. 6687. Presented at the Society of Photo-Optical Instrumentation Engineers (SPIE) Conference, p. 66870I (2007)

4. Serre, D., Deba, P., Koechlin, L.: Fresnel interferometric imager: ground-based prototype. Appl. Opt. 48, 2811-2820 (2009)

5. Faklis, D., Morris, G.M.: Broadband imaging with holographic lenses. Opt. Eng. 28(6), 592-598 (1989) 